IAC-18,E2,1,7,x46883

\title{
IMPACT PROBABILITY COMPUTATION FOR NEO RESONANT RETURNS THROUGH A POLYNOMIAL REPRESENTATION OF THE LINE OF VARIATIONS
}

\author{
Marcello Sciarra, Matteo Losacco, Daniele Santeramo, Pierluigi Di Lizia \\ Department of Aerospace Science and Technology, Politecnico di Milano, via la Masa 34, 20156, Milan, Italy \\ marcello.sciarra@mail.polimi.it
}

A differential algebra based representation of the Line of Variations for Near Earth Objects impact monitoring is presented in this paper. In this framework, the Line of Variations is described at the initial epoch by a high-order polynomial that is propagated forward in time. An Automatic Domain Splitting algorithm is embedded in the numerical integrator, in such a way that when the polynomials truncation error becomes too large, the line is split as many times as necessary to meet accuracy requirements. The Line of Variations is propagated forward in time until an intersection with a properly defined target plane occurs for all the generated subdomains. The projection of the subdomains onto the target plane allows to compute the impact probability by numerically integrating an associated one-dimensional probability density function. The proposed approach is applied to different test-cases to assess the performance of the method for the different possible shapes of the initial confidence region. Starting from a case of direct encounter, the technique is tested up to the case of a resonant return, in which the nonlinearities of the problem represent a critical aspect.

keywords: Line of Variations, Impact probability, Near Earth Asteroids, Resonant Return, Differential Algebra, Automatic Domain Splitting

\section{INTRODUCTION}

The increasing attention towards Space Situational Awareness (SSA) drives the development of efficient and reliable numerical techniques for many different applications in the field of astrodynamics. In particular, as more and more Near Earth Objects (NEO) are detected by the scientists, an efficient estimation of their impact probability with the Earth becomes a crucial requirement. If the observational campaign is short, the initial uncertainty about the nominal asteroid's state may be large and its numerical propagation in a nonlinear dynamics becomes computationally expensive. A critical case is represented by the phenomenon of resonant return: when an asteroid experiences a deep encounter with a major planet, the set of possible post-encounter orbits is enlarged by the encounter's nonlinearities and the post-encounter period may range between two values $P_{\min }$ and $P_{\max }$. All the rational numbers in this interval correspond to potential resonant returns: indeed, if the asteroid's period is $P=\frac{h}{k}$ years, then after $k$ revolutions of the asteroid and $h$ revolutions of the Earth, the two bodies may come to a collision. Due to the first encounter, the uncertainty region undergoes a strong expansion and the computational burden rises accordingly.

The two classical techniques adopted for nonlinear uncertainties propagation are Monte Carlo simulations and linearizations. While the former are very accurate but timeconsuming, the latter are faster yet provide low accuracy. The current state-of-the-art technique in this field is based on the idea of the Line of Variations (LOV), introduced for the first time by A. Milani in [4]. The LOV is a line of weakness in the orbit determination solution that offers the possibility to describe the behaviour of the six-dimensional uncertainty through a one-dimensional curve, exploiting its elongated shape. When the orbit of an asteroid is determined, a confidence region is associated to the nominal state: neglecting higher order terms, this uncertainty can be represented by a covariance matrix $\Gamma(X)$, being $X$ the six-dimensional orbital state. Calling $\sigma(X)$ the largest eigenvalue of $\Gamma(X)$ and $V_{1}(X)$ the corresponding eigenvector, the LOV is defined as the vector field that solves the differential equation:

$$
\frac{\mathrm{d} X}{\mathrm{~d} \sigma}=\sigma(X) V_{1}(X)
$$

In a linear approximation this simply reduces to the eigenvector corresponding to the major axis of the covariance ellipsoid $\Gamma(X)$.

Following the standard approach, as presented by Chesley and Chodas in [2], the LOV is sampled according to the chosen probability distribution, either uniform or Gaussian. Each generated sample is called virtual asteroid (VA) and has a certain probability to represent the true orbit. The VAs that directly impact the Earth take the name of virtual impactors (VI) and in the simplest approach the impact probability is computed as the ratio of VIs over VAs. Such approach does not consider all impacts that may occur for nearby off-LOV solutions and therefore some local analysis in the neighborhood of the close approaches must be performed. To perform this analysis, a target plane has to be exploited to eliminate the role of time: the b-plane (orthogonal to the incoming asymptote of the geocentric hyperbola) can be adopted if the nominal orbit enters the sphere of influence of the Earth. On the other hand, the modified target plane (MTP) is orthogonal to the closest approach point on the trajectoy and therefore includes larger nonlinearities in the numerical propagation. The MTP was introduced in 1999 by Milani and Valsecchi in [5 although the concept can be found earlier, see Chodas and Yeomans in [10].

After projecting the initial covariance onto the target plane at the collision epoch for the VIs, the impact probability (IP) can be computed by integrating a one-dimensional or two-dimensional probability density function (pdf), depending on the elongation of the confidence ellipse on the target 

permission and released to the IAF to publish in all forms.

plane that is by what amount the major axis $\Lambda$ is larger than the minor axis $w$ :

- if $\Lambda \gg w$ then $P_{I}=\frac{p\left(\sigma_{\Lambda}\right)}{\Lambda}$

- if $\Lambda>w$ then $P_{I}=\frac{p\left(\sigma_{\Lambda}\right) p\left(\sigma_{w}\right)}{\Lambda w} \pi r_{\oplus}^{2}$

The approach proposed in this paper is to move from a sampled description of the LOV to a polynomial representation of the curve, exploiting a differential algebraic framework to perform the propagation of the polynomially described curve. By propagating a continuous curve, one can avoid the issue of interpolating between the different samples and a very detailed description on the LOV evolution can be obtained.

The paper is organized as follows. Section II briefly introduces differential algebra (DA) and the automatic domain splitting (ADS) algorithm. Section III explains the numerical technique adopted to define the target plane, propagate the LOV until it intersects the plane and finally project the curve onto the MTP. In Section IV, the IP computation technique is presented through integration of the one-dimensional pdf. Section V shows the results from the application of the technique to a direct encounter test case. Section VI explains the issues indroduced by a resonant return and the technique adopted to overcome the problem. Section VII presents the issue of an enlarged initial confidence region and introduces a potential solution. Finally, Section VIII concludes the paper.

\section{DIFFERENTIAL ALGEBRA AND ADS}

Differential algebraic techniques were developed after the need to solve analytic problems with algebraic means. The theory of DA was developed for the first time by Joseph Liouville during the XIX century but the complete algebraic theory of the technique was presented by Ritt in [7]. The following overview is based on the description given by Martin Berz in [1].

Typically, in a computational environment, numbers take the place of functions and the algorithms are based on the pointwise functions evaluation. DA techniques try to increase the information that can be obtained from a function with respect to its mere evaluation. This additional information is provided by the implementation of an algebra of Taylor polynomials to which differentiation and integration can be easily added, thus obtaining a DA.

A relevant application of DA techniques arises for the integration of an ODE set, as explained in [3] in detail: in this framework, a high order polynomial expansion of the final integration state can be obtained with respect to the initial condition. This is achieved by substituting all the operations involved in the numerical integration scheme with the corresponding DA operations. In particular, if the initial polynomial represents the $n$ - $\sigma$ uncertainty region about the nominal state, its numerical propagation yields a polynomial description of the confidence region at the final integration time.

When the dynamics is highly nonlinear and the propagation time is long enough, a single polynomial expansion is not capable of providing an accurate description of the real confidence region evolution. To maintain a certain level of accuracy, an Automatic Domain Splitting (ADS) algorithm can be

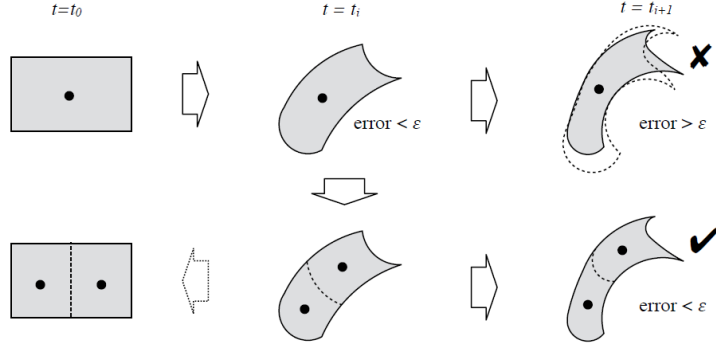

Fig. 1: Basic principle of Automatic Domain Splitting

implemented to support the simple DA propagation, see [9]. Such algorithm, whose working principle is sketched in Figure 1. estimates the truncation error of the polynomials over their own domain and decides wheather the truncation error is above or below a selected tolerance. When the estimated error is larger than the tolerance, the previous integration state (i.e. the last accurate state in the propagation history) is retrieved and split in two equal parts along a certain direction. This direction corresponds to the state variable along which the truncation error is estimated to be the largest. By dividing the domain by a factor 2 , the truncation error is reduced by a factor $2^{n+1}$, as explained in detail in [9]. The polynomials are then evaluated on the two generated subdomains and propagated independently until the truncation error overcomes again the selected tolerance. This procedure is repeated as many times as necessary, until a certain limit of splits $\mathcal{N}_{\max }$ is reached. Once the splitting tolerance tol $_{\text {split }}$ and $N_{\max }$ are defined, the procedure is fully automatic and guarantees an accurate nonlinear propagation of uncertainties. In this work, we have set $t_{\text {split }}=10^{-10}, \mathcal{N}_{\max }=16$ and DA polynomials expansion order equal to 12 .

The DA routines exploited in this work are implemented in the DACE software and more details on the numerical implementation can be found in [6]. The adopted numerical integration scheme is a DA version of a 7/8 Dormand-Prince (8th order solution for propagation, 7th order solution for step size control) Runge- Kutta integrator.

To better illustrate the application of ADS to ODE numerical integration, the propagation of an initial uncertainty within the Restricted Two Body Problem (R2BP) is now presented. Calling $\vec{r}$ and $\vec{v}$ the object's position and velocity vectors, the dynamics is given by:

$$
\dot{\vec{x}}=\left\{\begin{aligned}
\dot{\vec{r}} & =\vec{v} \\
\dot{\vec{v}} & =-\frac{\mu}{r^{3}} \vec{r}
\end{aligned}\right.
$$

where $\mu$ is the Sun gravitational parameter. The nominal initial conditions are set in such a way that the object starts moving from the pericenter of an orbit with eccentricity of 0.5 , lying on the ecliptic plane. The units are normalized and both the pericenter radius and $\mu$ are equal to 1 , thus leading to the following initial conditions:

$$
\vec{x}_{0}=\left\{\begin{aligned}
x & =1 \\
y & =0 \\
\dot{x} & =0 \\
\dot{y} & =\sqrt{1.5}
\end{aligned}\right.
$$




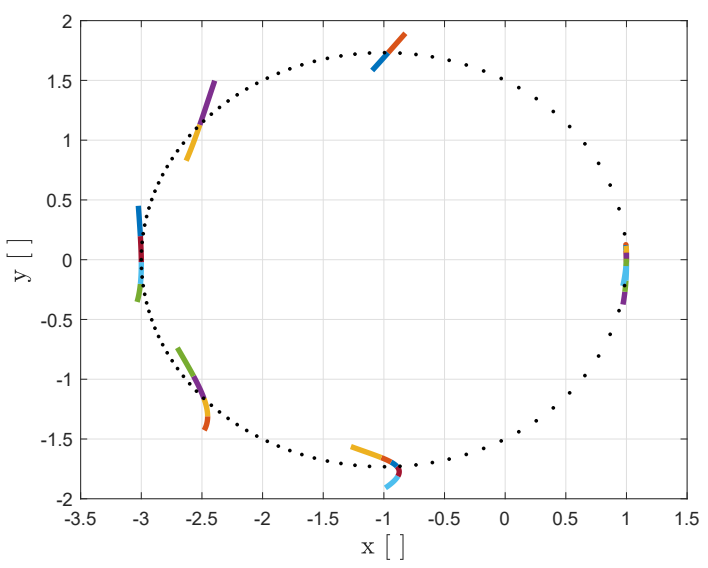

Fig. 2: Propagation of a y-axis uncertainty in the R2BP

An initial uncertainty of $\delta y= \pm 0.08$ is taken on the y-axis and propagated forward in time. The result is illustrated in Figure 2 in which the evolution of the uncertainty line is plot at different fractions of the orbital period. We can appreciate the effect of nonlinearities, which entails the need of an increasing number of splits of the line during the propagation.

\section{DA-BASED LINE OF VARIATIONS}

The Line of Variations is generated from the covariance matrix expressed in Cartesian coordinates. All the data for this analysis have been taken from the NEODyS database, that provides covariance matrices expressed in Equinoctial parameters. The adopted conversion technique is now explained. Calling $\vec{x}_{E}$ the orbital state expressed in Equinoctial parameters and $\vec{x}_{C}$ the Cartesian coordinates representation of the same state, the two states are related by a nonlinear coordinates transformation:

$$
\vec{x}_{C}=\mathcal{F}\left(\vec{x}_{E}\right)
$$

In a generic formulation the uncertainty region in the Equinoctial parameters space can be described by moments higher than just the mean and the covariance but in our case only the covariance matrix is given in the initial coordinates and only the covariance in the transformed coordinates is demanded. In the DA framework, the state in the original coordinates is written as:

$$
\left[\vec{x}_{E}\right]=\overrightarrow{\bar{x}}_{E}+\delta \vec{x}_{E}
$$

where $\vec{x}_{E}$ is the initial mean and the $\delta \vec{x}_{E}$ is the $1 \sigma$ variation. Applying the transformation $\mathcal{F}$ to $\overrightarrow{\bar{x}}_{E}$, yields the Taylor expansion of the final solution with respect to the deviations of the initial independent variable $\delta \vec{x}_{E}$ :

$$
\begin{aligned}
{\left[\vec{x}_{C}\right]=\mathcal{F}\left(\left[\vec{x}_{E}\right]\right)=\vec{x}_{C}+\mathcal{M}_{C}\left(\delta \vec{x}_{E}\right) } \\
\quad=\sum_{p_{1}+\cdots+p_{n} \leq k} c_{p_{1} \ldots p_{n}} \cdot \delta x_{1}^{p_{1}} \ldots \delta x_{n}^{p_{n}}
\end{aligned}
$$

where $\vec{x}_{C}$ is the zeroth-order term of the expansion map, $\mathcal{M}_{C}\left(\delta \vec{x}_{E}\right)$ is the Taylor map of the final state with respect to the initial state and $c_{p_{1} \ldots p_{n}}$ are the coefficients of the resulting Taylor polynomial. The Taylor series in the form of Equation 6 can be used to efficiently compute the propagated statistics by computing the $j$-th moment of the transformed pdf using a proper form of the $j$-th power of the solution map. The analytical expressions of the first two statistical moments for a generic scalar random variable $z$ are:

$$
\left\{\begin{array}{l}
\mu=E\{z\} \\
P=E\left\{(z-\mu)^{2}\right\}
\end{array}\right.
$$

where $\mu$ is the mean value, $P$ is the covariance and the expectation value of $z$ is:

$$
E\{z\}=\int_{-\infty}^{+\infty} z g(z) \mathrm{d} z
$$

The moments of the transformed pdf can be computed by applying the multivariate form of Equation 7 to the Taylor expansion in Equation 6. The result for the first two moments becomes:

$$
\left\{\begin{aligned}
\vec{\mu}_{i}= & E\left\{\left[\vec{x}_{C_{i}}\right]\right\}= \\
= & \sum_{p_{1}+\cdots+p_{n} \leq k} \vec{c}_{i, p_{1} \ldots p_{n}} \vec{E}\left\{\delta x_{1}^{p_{1}} \ldots \delta x_{n}^{p_{n}}\right\} \\
\vec{P}_{i j}= & E\left\{\left(\left[\vec{x}_{C_{i}}\right]-\mu_{i}\right)\left(\left[\vec{x}_{C_{j}}\right]-\mu_{j}\right)\right\}= \\
= & \sum_{p_{1}+\cdots+p_{n} \leq k, \vec{c}_{i, p_{1} \ldots p_{n}} \vec{c}_{j, p_{1} \ldots p_{n}}} \\
& q_{1}+\cdots+q_{n} \leq k \\
& E\left\{\delta x_{1}^{p_{1}+q_{1}} \ldots \delta x_{n}^{p_{n}+q_{n}}\right\}
\end{aligned}\right.
$$

where $\vec{c}_{i, p_{1} \ldots p_{n}}$ are the Taylor coefficients of the Taylor polynomial describing the $i$-th component of $\left[\vec{x}_{C}\right]$. The expectations in Equation 9 are computed through the Isserlis' formula. Full details on the method and its generalization can be found in [8].

When the covariance matrix in Cartesian coordinates is known, its eigenvalues and eigenvectors can be computed and the LOV can be initialized, according to the linear approximation definition presented in Section I. Considering the eigenvalues and eigenvectors as sorted in ascending order of magnitude (i.e. the last component corresponds to the major axis), the LOV is first initialized in the space of the eigenvectors as:

$$
X_{\mathrm{EIG}}=\left[\begin{array}{llllll}
0 & 0 & 0 & 0 & 0 & \Lambda \cdot \delta x_{6}
\end{array}\right]^{T}
$$

where $\Lambda$ is a scaling factor for the DA polynomial. If the desired analysis covers the $\pm 3 \sigma$ interval, such factor becomes $\Lambda=3 \sqrt{\lambda_{6}}$. The real LOV is finally obtained by rotating $X_{\text {EIG }}$ through the eigenvectors matrix $\mathrm{U}$ and summing up the nominal state:

$$
X_{\mathrm{LOV}}=X_{0}+U \cdot X_{\mathrm{EIG}}
$$

Before propagating the generated LOV, the target plane has to be defined and we decided to adopt the MTP. To define the plane, a propagation of the nominal state is performed until the condition of minimum geocentric distance is met. Since the propagation spans many revolutions of the asteroid, this condition (i.e. $\rho=\vec{r}_{\mathrm{GEO}} \cdot \vec{v}_{\mathrm{GEO}}=0$ ) is verified several times. In this version of the tool, we decided to work with a given estimated collision epoch and therefore the condition above is considered to be met only if it occurs inside a certain search timespan of \pm 100 days around the given collision 
epoch. When the scalar product $\rho$ changes sign during the propagation, a bisection routine is started to identify the exact zero-scalar-product condition and subsequently define the MTP reference frame.

When the MTP is defined, the full ADS-based propagation of the LOV can be performed within an N-body dynamics with relativistic correction. The dynamics, taken from [9], is:

$$
\begin{aligned}
& \ddot{\vec{r}}=G \sum_{i} \frac{m_{i}\left(\vec{r}_{i}-\vec{r}\right)}{r_{i}^{3}}\left\{1-\frac{2(\beta+\gamma)}{c^{2}} G \sum_{j} \frac{m_{j}}{r_{j}}-\right. \\
& \frac{2 \beta-1}{c^{2}} G \sum_{j \neq i} \frac{m_{j}}{r_{i j}}+\frac{\gamma|\dot{\vec{r}}|^{2}}{c^{2}}+\frac{(1+\gamma)\left|\dot{\vec{r}}_{i}\right|^{2}}{c^{2}}- \\
& -\frac{2(1+\gamma)}{c^{2}} \dot{\vec{r}} \cdot \dot{\vec{r}}_{i}-\frac{3}{2 c^{2}}\left[\frac{\left(\vec{r}-\vec{r}_{i}\right) \cdot \dot{\vec{r}}_{i}}{r_{i}}\right]^{2}+ \\
& \left.+\frac{1}{2 c^{2}}\left(\vec{r}_{i}-\vec{r}\right) \cdot \ddot{\vec{r}_{i}}\right\}+G \sum_{i} \frac{m_{i}}{c^{2} r_{i}} \cdot\left\{\frac{3+4 \gamma}{2} \ddot{\vec{r}}+\right. \\
& \left.+\frac{\left\{\left[\vec{r}-\vec{r}_{i}\right] \cdot[(2+2 \gamma) \dot{\vec{r}}\right.}{r_{i}^{2}}-\frac{\left.\left.(1+2 \gamma) \dot{\vec{r}}_{i}\right]\right\}\left(\dot{\vec{r}}-\dot{\vec{r}}_{i}\right)}{r_{i}^{2}}\right\}
\end{aligned}
$$

During the propagation, the initial LOV is split several times, particularly if a deep encounter with a major planet occurs. For each of the generated subdomains, the intersection with the target plane close to the collision epoch shall be determined. A large number of numerical conditions has to be applied to avoid all possible issues. More specifically:

- the intersection must occur inside the search timespan.

- the intersection must occur in the same direction of the nominal state.

- when a subdomain reaches the MTP or the final integration time and the following one is initialized for propagation, the sign of the scalar product at the two states may be opposite. Therefore the algorithm may detect a fictitious crossing of the MTP that must be avoided.

- when a subdomain experiences a split while it lies across the MTP, particular care has to be adopted to avoid that one of the two generated subdomains misses the intersection with the plane.

To solve all these issues, when a subdomain is initialized for propagation its propagation is forced to start with a fixed stepsize of $10^{-8}$. This guarantees a better control on the presented numerical issues: exploiting the knowledge of the stepsize allows to either include or exclude the first steps of a subdomain's propagation from the MTP intersection checking.

The correct intersections of the MTP are identified by checking at every integration step the following conditions:

$$
\left\{\begin{array}{l}
t_{k}>\left(t_{\mathrm{IMP}}-100 \mathrm{dd}\right) \\
\left(\vec{r}_{\mathrm{GEO}_{k}} \cdot \vec{V}\right) \cdot\left(\vec{r}_{\mathrm{GEO}_{k-1}} \cdot \vec{V}\right)<0 \\
\vec{r}_{\mathrm{GEO}_{k}} \cdot \vec{V}>0 \\
|h|>2 \cdot 10^{-8} \\
t_{k-1}<\left(t_{\mathrm{IMP}}-\Delta t_{\text {max }}\right)
\end{array}\right.
$$

$$
\left\{\begin{array}{l}
t_{k}>\left(t_{\mathrm{IMP}}-100 \mathrm{dd}\right) \\
\vec{r}_{\mathrm{GEO}_{k}} \cdot \vec{V}>0 \\
|h|<2 \cdot 10^{-8} \\
\left|\vec{r}_{\mathrm{GEO}_{k-1}} \cdot \vec{V}\right|>10^{-10} \\
\vec{r}_{\mathrm{GEO}_{k}}\left|-1.0 \cdot \vec{r}_{\mathrm{GEO}_{k}}\right|_{1.0}<0 \\
t_{k-1}<\left(t_{\mathrm{IMP}}-\Delta t_{\text {max }}\right)
\end{array}\right.
$$

In the equations above, $t_{\mathrm{IMP}}$ represents the given collision epoch, $h$ is the current stepsize, $k$ subscript refers to the $k$ th integration step, $k-1$ to the $(k-1)$-th step and $\Delta t_{\max }$ is a fixed time interval that the propagator cannot span with a single integration step. Note that the $4^{\text {th }}$ condition of Equation 13 and the $3^{\text {rd }}$ condition of Equation 14 are complementary and therefore the two sets cannot be satisfied simultaneously. If one of the two sets is fully satisfied, a Newton's method can be triggered to approach the plane.

When the propagation of all the subdomains is over, those that intersected the MTP have to be projected onto the plane. Instead of projecting along the direction normal to the plane, the projection is performed along the direction of the velocity vector in order to better approximate the dynamical path that would lead each point of the subdomain to the MTP. DA can be exploited to guarantee an accurate projection: By considering that we know the polynomial expansion of the asteroid's velocity along each subdomain, if we perform a full DA-based projection, we can project each point of the subdomain along its own velocity vector. The final position vector:

$$
\vec{r}=x \vec{i}+y \vec{j}+z \vec{k}
$$

needs to be expressed as:

$$
\vec{r}=c_{1} \vec{R}+c_{2} \vec{v}+c_{3} \vec{Z}
$$

where $\vec{v}$ is the polynomial expression of the box velocity vector while $\vec{R}$ and $\vec{Z}$ are the in-plane unit vectors of the MTP frame. The goal of this projection is to find the coefficients $c_{1}$ and $c_{3}$ that will represent the in-plane coordinates. The $c_{2}$ coefficient that represents the out-of-plane coordinate is implicitly set to zero. Computing the scalar product of Equation 16 with $\vec{R}, \vec{v}$ and $\vec{Z}$ respectively, the result is:

$$
\begin{aligned}
\vec{r} \cdot \vec{R} & =c_{1} \vec{R} \cdot \vec{R}+c_{2} \vec{v} \cdot \vec{R}+c_{3} \vec{Z} \cdot \vec{R}= \\
& =c_{1}+c_{2} \vec{v} \cdot \vec{R} \\
\vec{r} \cdot \vec{v} & =c_{1} \vec{R} \cdot \vec{v}+c_{2} \vec{v} \cdot \vec{v}+c_{3} \vec{Z} \cdot \vec{v}= \\
& =c_{1} \vec{R} \cdot \vec{v}+c_{2} v^{2}+c_{3} \vec{Z} \cdot \vec{v} \\
\vec{r} \cdot \vec{Z} & =c_{1} \vec{R} \cdot \vec{Z}+c_{2} \vec{v} \cdot \vec{Z}+c_{3} \vec{Z} \cdot \vec{Z}= \\
& =c_{2} \vec{v} \cdot \vec{Z}+c_{3}
\end{aligned}
$$

The left-hand sides are known and are called $A=\vec{r} \cdot \vec{R}, B=$ $\vec{r} \cdot \vec{v}$ and $C=\vec{r} \cdot \vec{Z}$ for the sake of notation. Furthermore we can call $\cos \alpha=\vec{v} \cdot \vec{R}$ and $\cos \beta=\vec{v} \cdot \vec{Z}$ and since the whole procedure has been carried out with the normalized velocity vector it follows that $v^{2}=1$. The simplified system that 

permission and released to the IAF to publish in all forms.

needs to be solved becomes:

$$
\begin{aligned}
& A=c_{1}+c_{2} \cos \alpha \\
& B=c_{1} \cos \alpha+c_{2}+c_{3} \cos \beta \\
& C=c_{2} \cos \beta+c_{3}
\end{aligned}
$$

This system can be easily solved to find the expression of the three coefficients $c_{1}, c_{2}$ and $c_{3}$. Working within DA, all the elements in the expressions above are polynomials and therefore the obtained coefficients are still polynomials. Since the procedure is carried out not only with normalized $\vec{v}$ but also with normalized $\vec{r}$, the obtained coefficients need to be multiplied by $|\vec{r}|$ to obtain the correct scaled MTP coordinates that will be:

$$
\mathcal{T}_{\vec{r}_{M T P}}=\left[\begin{array}{ll}
\mathcal{T}_{c_{1}}|\vec{r}| & \vec{R} \\
\mathcal{T}_{c_{3}}|\vec{r}| & \vec{Z}
\end{array}\right]
$$

Together with allowing for a more accurate projection, DA gives the possibility to have a polynomial expansion of the MTP position vector, which means that the full Line of Variations is described as a one-dimensional polynomial expression in MTP coordinates.

This has a key role in eliminating the time variable from the subsequent analysis. Indeed, during the propagation the confidence region very quickly spreads along the trajectory, thus becoming an anomaly uncertainty. For this reason, all the different subdomains reach the target plane at very different times but in any case very close to their actual point of closest approach to the Earth. Therefore, by analyzing the projection of the LOV onto the MTP, the impact probability can be properly computed.

\section{1D IMPACT PROBABILITY COMPUTATION}

Once the propagation and the projection of the LOV have been performed, the LOV is represented onto the MTP by a series of subdomains of different dimensions, some of them having a geocentric distance lower than the Earth radius. In Figure 3 a sketch of the LOV on the MTP is illustrated: the different subdomains that constitute the LOV are highlighted with different colours and the impacting ones are dashed. Considering a one-dimensional pdf associated to the LOV, the impact probability between the asteroid and the Earth can be computed by integrating the pdf over the impacting subdomains. Different

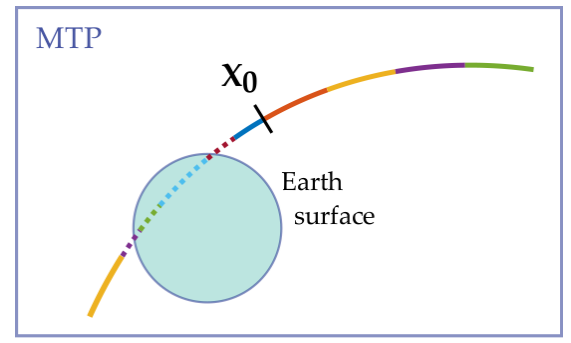

Fig. 3: Sketch of the LOV projected on the MTP: dashed subdomains are the impactors and they are considered in the IP computation

approaches can be exploited to perform the computation:
- Integration of the propagated pdf - The nonlinear dynamics enlarges the asteroid's confidence region, thus stretching the LOV. If a single subdomain is considered, the ratio of its length at the final and initial epoch provides a stretching factor $\Lambda^{1}$ This stretching factor can be used to propagate the pdf associated to that subdomain. By multiplying it by the length of the subdomain at the final epoch, the impact probability can be computed:

$$
I P=\frac{p(\sigma)}{\Lambda} l
$$

- DA-based Monte Carlo - In a DA framework, a polynomial expansion of the final integration state with respect to the initial conditions is given, as explained in Section II. Therefore, the final state corresponding to each point of the initial LOV can be retrieved by just evaluating this polynomial map. Therefore the initial LOV can be sampled according to the chosen pdf and the impact probability can be computed as the ratio of the number of samples whose corresponding final state impacts with the Earth and the total number of samples:

$$
I P=\frac{N_{\oplus}}{N}
$$

- Integration of the pdf over the initial domain-Once the impacting subdomains are detected, the impact probability can be computed by simply integrating the pdf associated to the initial LOV over the portion of the line that collides with the Earth. This integration can be performed analytically, exploiting a DA-based numerical integration scheme, or algebraically by just assuming a constant pdf over each subdomain.

The first method guarantees the possibility to perform a target plane analysis, if needed. On the other hand the DA-based Monte Carlo is very simple and accurate provided that the final polynomial map is accurate enough. As this condition is not guaranteed in the case of a highly nonlinear dynamics and a target plane analysis in its standard sense (see [2]) is not performed in this work, the third method is adopted. A Gaussian pdf is associated to the initial LOV in the form:

$$
\operatorname{pdf}(x)=\frac{1}{\sqrt{2 \pi} \sigma} e^{-\frac{(x-\mu)^{2}}{2 \sigma^{2}}}
$$

The mean value $\mu$ is equal to zero in this case as the pdf is centered in the expansion point of the LOV. In the DA framework the DA independent variable $\delta$ goes from -1 to +1 in the corresponding real interval $[-3 \sigma,+3 \sigma]$. To compute the probability density at a generic coordinate $x=q \sigma$ (that is $\delta=$ $\frac{q \sigma}{3 \sigma}=\frac{q}{3}$ ) the exponent of Equation 26 expression becomes:

$$
-\frac{(x-\mu)^{2}}{2 \sigma^{2}}=-\frac{(q \sigma)^{2}}{2 \sigma^{2}}=-\frac{1}{2} q^{2}=-\frac{1}{2}(3 \delta)^{2}
$$

${ }^{1}$ The same symbol is used here as for the major axis of the confidence ellipse on the MTP, seen in Section I. Indeed there is a strong correlation between the two parameters. If we consider the initial 6D confidence region and project it onto the MTP to become a 2D ellipse, in a first-order approximation its semi-major axis is exactly the propagated and projected LOV 
and the pdf expression that can be adopted within the DA framework is:

$$
\operatorname{pdf}(x)=\frac{1}{\sqrt{2 \pi} \sigma} e^{-\frac{1}{2}(3 \delta)^{2}}
$$

In a first approximation, the subdomains whose center or one of the extrema lie below the Earth surface can be considered as fully impacting. A refinement can be offered by the possibility to find the exact portion of the LOV that intersects the Earth surface, increasing the precision in the computed IP. Making reference to Figure 4 , the subdomains are classified as:

- Transition - Moving from left to right along the LOV, the last red subdomain before one or more blue subdomain and the first blue subdomain after one or more red subdomains. For these subdomains the exact intersection shall be found.

- Internal - Subdomains whose center lies below the Earth surface and are not close to the intersection. They are considered as full impactors.

- External - Subdomains whose center lies above the Earth surface and are not close to the intersection. They are excluded from the IP computation.

For the transition subdomains, the intersection with the Earth surface can be computed by numerically finding the root of the function:

$$
f=\left\|\vec{r}_{\mathrm{MTP}}\right\|-R_{\oplus}
$$

where $R_{\oplus}$ is the Earth radius and $\left|\vec{r}_{\mathrm{MTP}}\right|$ is the polynomial expansion of the norm of the asteroid's position on the MTP. Finding the exact intersection between the LOV and the Earth surface allows to define two coefficients $\mathcal{B}_{i}$ and $\mathcal{D}_{i}$ that are used to refine the IP computation. Before applying the bisection method, the presence of an actual zero has to be checked. The function is evaluated at the extrema and at the center point and the three values are called for simplicity:

$$
A=f(+1) \quad B=f(0) \quad C=f(-1)
$$

Calling $\mathcal{Z}$ the root of the function, the different possibilities arise for the assignment of the coefficients $\mathcal{B}_{i}$ and $\mathcal{D}_{i}$

1. $\mathbf{A} \cdot \mathbf{B}<\mathbf{0}$ Search for $\mathcal{Z}$ between 0 and +1 .

a) $\mathbf{A}<\mathbf{0}$

$$
\begin{aligned}
& \mathcal{B}=0.5 \cdot(1-|\mathcal{Z}|) \\
& \mathcal{D}=0.5 *(1.0+\mathcal{Z})
\end{aligned}
$$

b) $\mathbf{A}>\mathbf{0}$

$$
\begin{aligned}
& \mathcal{B}=0.5+0.5|\mathcal{Z}| \\
& \mathcal{D}=0.5 *(-1.0+\mathcal{Z})
\end{aligned}
$$

2. $\mathbf{C} \cdot \mathbf{B}<\mathbf{0}$ Search for $\mathcal{Z}$ between -1 and 0 .

a) $\mathbf{C}<\mathbf{0}$

$$
\begin{aligned}
& \mathcal{B}=0.5 \cdot(1-|\mathcal{Z}|) \\
& \mathcal{D}=0.5 *(-1.0+\mathcal{Z})
\end{aligned}
$$

b) $\mathbf{C}>\mathbf{0}$

$$
\mathcal{B}=0.5+0.5|\mathcal{Z}|
$$

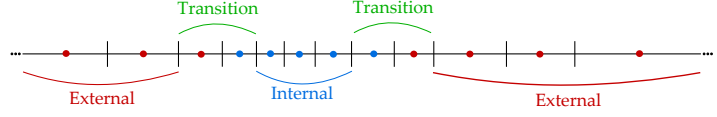

Fig. 4: Principle of the IP refinement

$$
\mathcal{D}=0.5 *(1.0+\mathcal{Z})
$$

3. $\mathbf{A}<\mathbf{0} \& \mathbf{B}<\mathbf{0} \& \mathbf{C}<\mathbf{0}$

$$
\begin{aligned}
& \mathcal{B}=1 \\
& \mathcal{D}=0
\end{aligned}
$$

4. $\mathbf{A}>\mathbf{0} \& \mathbf{B}>\mathbf{0} \& \mathbf{C}>\mathbf{0}$

$$
\begin{aligned}
& \mathcal{B}=0 \\
& \mathcal{D}=0
\end{aligned}
$$

When this procedure is over, the impact probability is computed through this formula:

$I P=\sum_{i} \frac{1}{\sqrt{2 \pi} \sigma} \exp \left\{-\frac{1}{2}\left[\left(c_{i}+\mathcal{D}_{i} \cdot 0.5 w_{i}\right) \sigma\right]^{2}\right\} \cdot 3 \sigma \mathcal{B}_{i} w_{i}$

in which $c_{i}$ and $w_{i}$ represent the center and the width of each subdomain with respect to the original domain, respectively. $\mathcal{D}_{i}$ defines by what ammount the point of evaluation of the pdf has to be shifted and $\mathcal{B}_{i}$ represents the portion of the subdomain that impacts with the Earth and therefore multiplies the pdf.

\section{ASTEROID 2010 RF12 - DIRECT ENCOUNTER}

The presented technique is now applied to a test case with a potential direct encounter collision, meaning that during the propagation no deep encounter occurs between the initial epoch and the collision epoch. Asteroid 2010 RF12 has a very elongated initial confidence region with a ratio of $3.8 \cdot 10^{4}$ between the largest and the second eigenvalue of the initial Cartesian covariance matrix, from now on called eigenvalues ratio $\chi$. Due to the high eigenvalues ratio, the LOV is expected to provide a very good approximation of the behaviour of the full confidence region. This asteroid, whose initial conditions at March 2018 have been taken from the NEODyS database, presents a potential direct collision in 2095.

Figure 5 shows the projection of the LOV onto the MTP, in which the low nonlinearity of the dynamics due to the absence of deep encounters is highlighted by the straight shape of the line. The subdomains (represeted by the different colours) get smaller and smaller getting closer to the planet (the small black dot close to the right end of the LOV) and, inside the Earth disk (in Figure 6 the detail is depicted) they become extremely small. Table 1 presents the IP computation for this test case, together with the reference value taken from the NEODyS database. It can be observed that the accuracy of the result is extremely high and the computational time of about 17 minutes is satisfactory, considering that the collision occurs in 2095, thus requiring about 80 years of numerical propagation. 
$69^{\text {th }}$ International Astronautical Congress, Bremen, Germany. Copyright $(2) 2018$ by Politecnico di Milano. Published by the IAF, with permission and released to the IAF to publish in all forms.

\begin{tabular}{ccccc}
\hline order & subdomains & comp. time [s] & IP & IP $_{\text {NEODyS }}$ \\
\hline 12 & 91 & 1017 & 0.05943 & 0.06 \\
\hline
\end{tabular}

Tab. 1: 2010 RF12 impact probability computation. The computational time is the running-time on an an AMD Opteron 6376 processor with a total of 64 cores @ 2.3 $\mathrm{GHz}$ and 256 Gbytes of RAM.

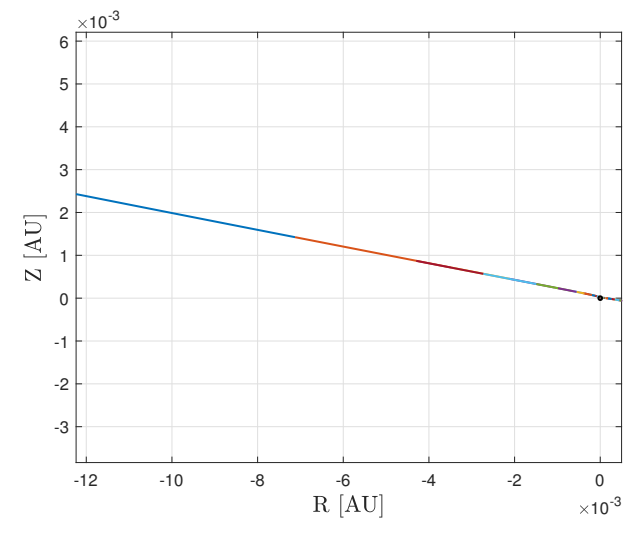

Fig. 5: 2010 RF12: LOV projection onto the MTP

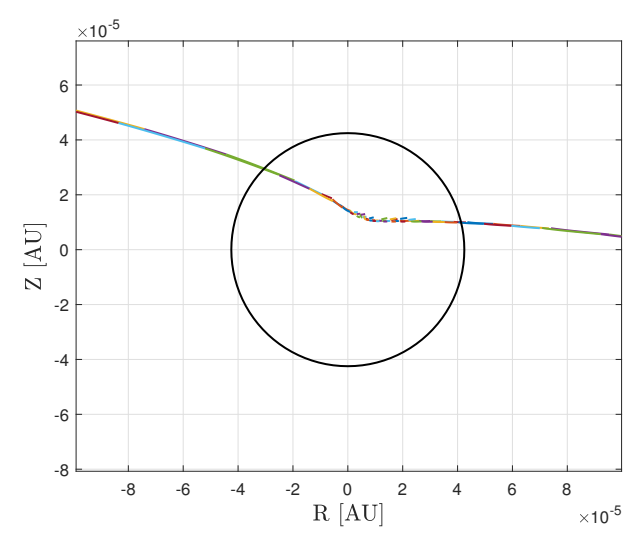

Fig. 6: 2010 RF12: detail of the LOV projection onto the MTP close to the Earth disk

\section{ASTEROID APOPHIS - RESONANT RETURN}

A resonant return introduces relevant complexity in the computation. To present this condition, the case of Apophis is introduced. When first observed, the asteroid was estimated to have a very high impact probability with the Earth in 2029. When this collision was ruled out, the 2029 deep encounter still accounted for a possible resonant return collision in 2036. Further observations ruled out also this collision. The initial condition used for this analysis were given by G. Tommei (private communication) and they are relative to a 2004-2005 observational campaign that includes the possibility of a 2036 collision. In this case we have $\chi=5 \cdot 10^{2}$, which is lower than the previous case but still describes an elongated initial confidence region. Indeed, the nonlinearities due to the deep planetary encounter critically enlarges the confidence region and the LOV loses the straightness it presented in the previous case as evident in Figure 7 . These nonlinearities induce a very high number of splits that very likely reaches the maxi-

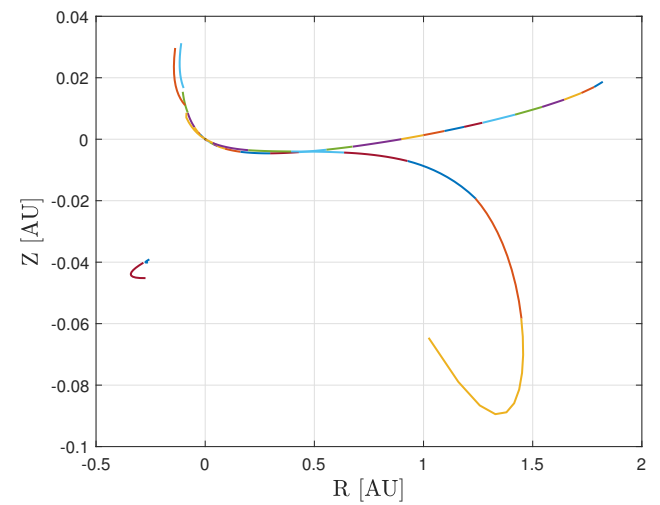

Fig. 7: Apophis: LOV projection onto the MTP

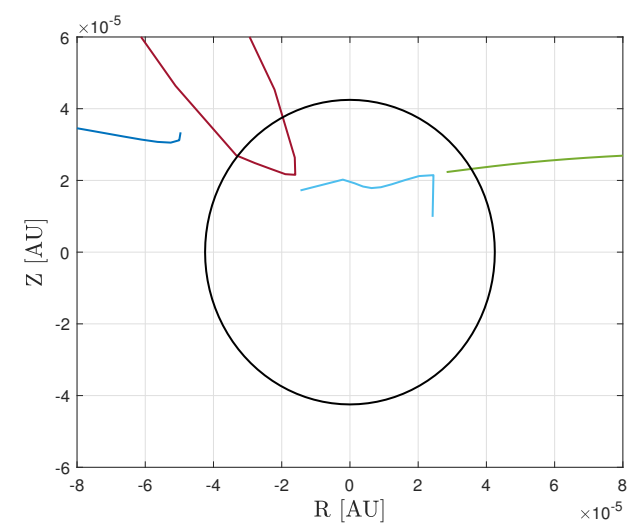

Fig. 8: Apophis: detail of the LOV projection onto the MTP close to the Earth disk

mum number of splits $\mathcal{N}_{\max }$. If this happens, the final polynomial map is not accurate and therefore the computation of the impact probability is not reliable. To better illustrate this issue, Figure 8 shows the projection of the portion of the LOV close to the Earth disk. It is evident that the inner subdomains (in red and light blue) are completely inaccurate due to the nonlinearitis and they could not be split further.

A solution to this problem is mandatory to guarantee a satisfactory result for asteroids with a resonant return condition. To achieve this, a refining routine is proposed:

1. After the first propagation is over, the subdomains (either accurate or inaccurate) whose center lies below the Earth surface are identified.

2. Classify the subdomains as Internal, External or Transition subdomains.

3. Internal subdomains are considered full impactors even if they are inaccurate in order to significantly reduce the computational burden.

4. Consider the Transition subdomains and retrieve the last accurate state within their propagation history.

5. Initialize a new ADS-based propagation of these subdomains that allows to further split the sudomains and to obtain an accurate polynomial map at least close to the Earth surface. 

permission and released to the IAF to publish in all forms.

6. Identify the exact intersection between the LOV and the Earth disk to compute an accurate IP.

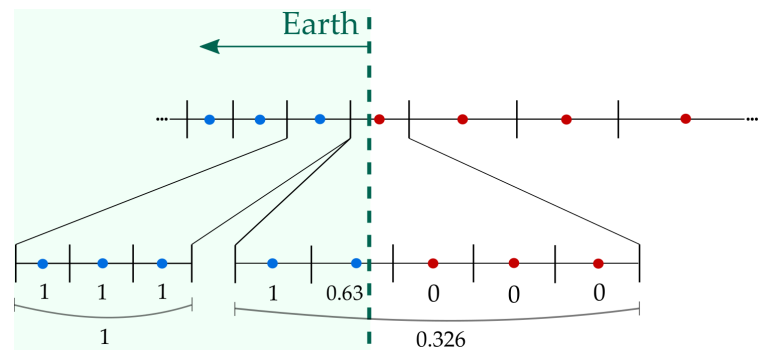

Fig. 9: Basic principle of subdomains repropagation

The last item shall be discussed in more detail. Making reference to Figure 9 , the center points of the blue subdomains lie below the Earth surface and the center points of the red subdomains lie above the surface. Moving from left to right, the last blue subdomain and the first red one constitute the transition subdomains, as the intersection between LOV and Earth surface may lie in either one or the other. These subdomains are repropagated and a simulation of what the outcome of this procedure might be is presented in the bottom part of the same sketch: several sub-subdomains are generated from each of the two initial subdomains. By applying a procedure similar to the one applied to the first propagation, the exact intersection can be identified inside one of the sub-subdomains. A coefficient is associated to each sub-subdomain, representing its portion that lies below the surface. These coefficients, together with the knowledge of the dimension of each subsubdomain, are used to define the coefficient $\mathcal{B}_{i}$ associated to the $i$-th original subdomain ( 0.326 in the Figure).

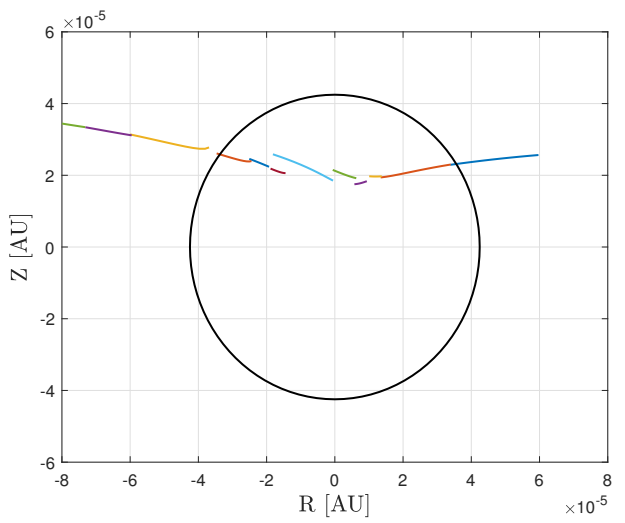

Fig. 10: Apophis: detail of the LOV projection onto the MTP close to the Earth disk

The outcome of the repropagation routine for Apophis is evident in Figure 10 The inaccurate subdomains are repropagated and this grants a better description of the LOV. Some subdomains inside the disk are detached from the rest of the LOV: this is not due to inaccuracy of the polynomials but rather to the projection routine. Indeed, the propagation of the subdomains that have a collision with the Earth is stopped and they are directly projected onto the MTP even if their center does not lie on the plane. This introduces an error that can

\begin{tabular}{ccccc}
\hline order & subdom. & comp. time $[\mathrm{s}]$ & $\mathrm{IP}$ & $\mathrm{IP}_{\mathrm{REF}}$ \\
\hline 12 & 130 & 1474 & $3.0134 \cdot 10^{-5}$ & $3 \cdot 10^{-5}$ \\
\hline
\end{tabular}

Tab. 2: Apophis impact probability computation

be considered to be quite negligible to the purpose of IP computation. The outcome of the algorithm is presented in Table 2 and also in this case the computed value is very close to the reference value.

VII. LOW EIGENVALUES RATIO AND 2D METHOD

The previous sections have shown two test cases in which the LOV is able to approximate the behaviour of the full confidence region and the computed impact probability is accurate. If we consider the initial $6 \mathrm{D}$ uncertainty ellipsoid, in general the VIs are gathered in a certain region, more or less elongated and variously distributed inside the ellipsoid. If this region lies across the LOV, i.e. the major axis, then the onedimensional LOV technique is capable of approximating the impact probability of all the VIs by reducing the analysis on a single direction. If the confidence region is very elongated this is very likely to be true, while this assumption may fail if the uncertainty ellipsoid is rather enlarged that is if the first two eigenvalues are about the same order of magnitude. This condition is witnessed by the eigenvalues ratio $\chi$, that assumes a key role in this analysis.

We have seen two cases in which the one-dimensional technique is capable of providing an accurate IP value. Now we want to assess the reliability of the method for lower values of the eigenvalues ratio. Consider asteroid 2016 LP10: it has an eigenvalues ratio of about 8 , that means the initial confidence region is extremely enlarged. The application of the technique presented so far yields $I P=0$ even if the impact probability according to the NEODyS database is $4.1 \cdot 10^{-5}$. This means that the VIs in this case lie in a region fully outside of the LOV and therefore the one-dimensional technique fails. Indeed, observing Figure 11 we can appreciate how the LOV does not collide with the Earth (the black dot close to the blue subdomain on the left). The classical LOV technique exploits a projection onto the MTP of the covariance relative to each VA in order to perform an off-LOV analysis. Yet, our analysis is carried out starting with a determined orbit and only one covariance matrix is known for the whole distribution. For this reason an off-LOV analysis cannot be performed and another technique needs to be applied. The easiest approach exploits the possibility to increase the number of variables of the polynomials, by considering the first two eigenvalues of the covariance matrix in the analysis.

Before presenting the two-dimensional approach, it is important to provide some hints on when the two methods have to be applied. From additional analysis, the following guidelines have been identified:

- $\chi>10^{4}$ the eigenvalues ratio is large enough that the one-dimensional technique can be deemed reliable.

- $\chi<10^{2}$ the eigenvalues ratio is too low to rely on the one-dimensional technique and a multi-dimensional technique has to be adopted. 


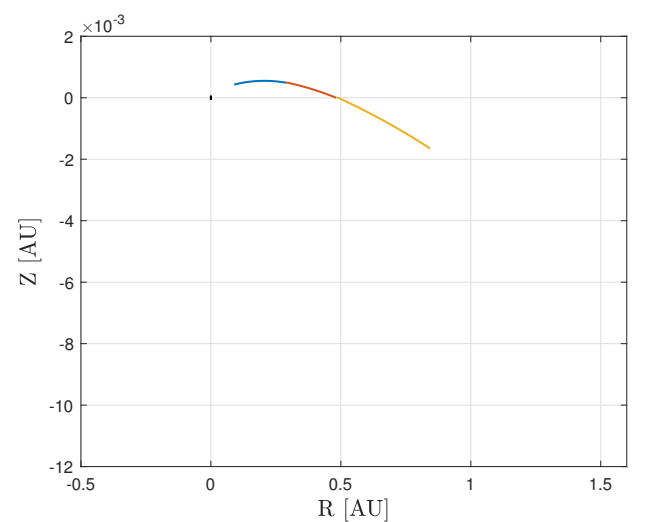

Fig. 11: 2016 LP10: LOV projection onto the MTP

- $\chi \approx 10^{2}-10^{3}$ the eigenvalues ratio has an intermediate value and this is the most critical case. Two possibilities arise:

$\mathbf{I P}=\mathbf{0}$ in this case a multi-dimensional technique has to be adopted to exclude the possibility of any offLOV impactors.

IP $\neq \mathbf{0}$ in this case the computed impact probability is in general reliable, even if many VIs lie off-LOV.

This last consideration is particularly interesting and requires some additional analysis to be fully explained. Consider a case in which the eigenvalues ratio is intermediate, such as asteroid 2017 RH16 for which $\chi=2.95 \cdot 10^{3}$. We can perform a one-dimensional propagation, followed by a two-dimensional propagation and for both propagations the impactors are identified. Figure 12 shows the 2D impactors (in blue) together with the LOV (the red line) and the 1D impactor (in black) on the plane of the first two eigenvectors. We can observe that the $2 \mathrm{D}$ impactors lie almost orthogonally to the LOV and this provides an explaination on how the one-dimensional technique is capable of providing a quite accurate impact probability $\left(1.04 \cdot 10^{-3}\right.$ versus $1.45 \cdot 10^{-3}$ as given from NEODyS) even if most VIs lie outside the LOV. Indeed, if we integrate a one-dimensional pdf we are implicitely integrating the other dimensions from $-\infty$ to $+\infty$. More specifically, if we consider the $1 \mathrm{D}$ impactor, this is equivalent to assuming as impactor the full line orthogonal to the LOV and crossing the line at the quote of the 1D impactor. Since the real VIs are almost perpendicular to the LOV, the two outputs are very similar to each other, thus justifying the adoption of a one-dimensional reduction of the problem.

When the one-dimensional LOV approach cannot be assumed to be reliable, a two-dimensional technique can be adopted. This is achieved by just observing that in the DA framework we can work with polynomials in $n$-variables. Moving from one to two variables polynomials, the onedimensional LOV can be substituted by a sort of plane of variations that, as before, is initialized in the space of the eigenvectors as:

$$
\mathbf{x}_{\mathrm{EIG}}=\left[\begin{array}{llllll}
0 & 0 & 0 & 0 & \Lambda_{5} \cdot \delta x_{5} & \Lambda_{6} \cdot \delta x_{6}
\end{array}\right]^{T}
$$

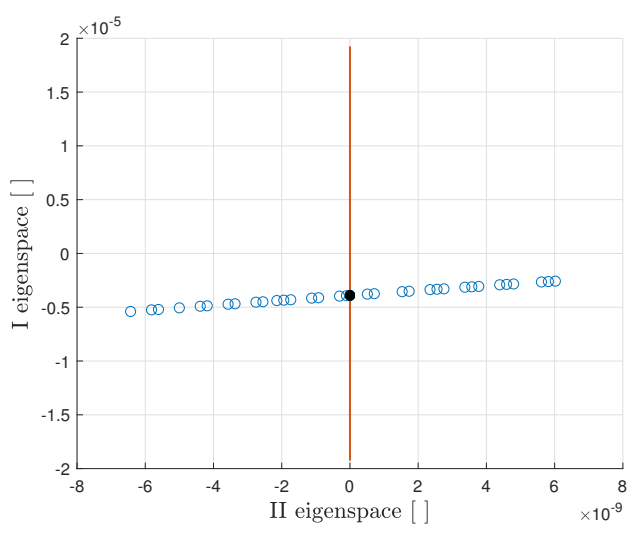

Fig. 12: 1D and 2D impactors for 2017 RH16

If we are performing $\mathrm{a} \pm 3 \sigma$ analysis, the scaling factors are:

$$
\Lambda_{5}=3 \sqrt{\lambda_{5}} \quad \text { and } \quad \Lambda_{6}=3 \sqrt{\lambda_{6}}
$$

$\mathbf{x}_{\text {EIG }}$ needs to be rotated in the real Cartesian space as done in Equation 11 and the result of this procedure will be a $6 \mathrm{D}$ vector whose components are in this case six polynomials in two variables.

During the propagation, the two-dimensional confidence region quickly spreads along the anomaly, as shown in Figure 13 for asteroid 2016 LP10, yielding once again sort of stripe on the MTP. It can be seen that in this type of propagation the subdomains are much more subject to deformation after reaching $\mathcal{N}_{\text {max }}$. For the sake of visual clarity, the polynomials are evaluated with a first order approximation to avoid that the high-order terms generate an unreadable figure.

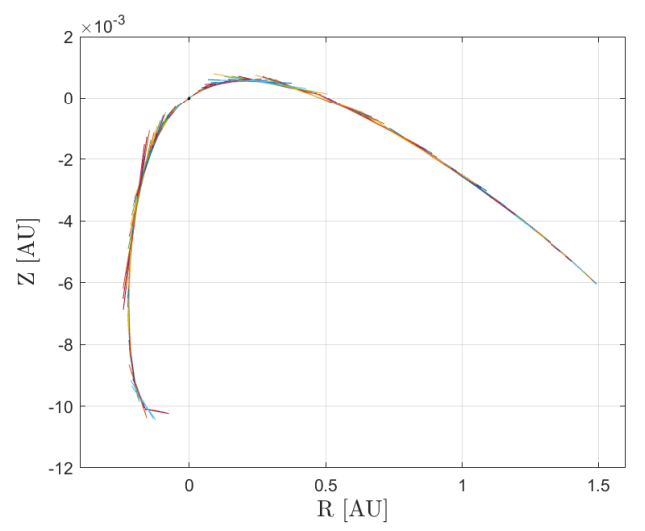

Fig. 13: 2016 LP10 2D confidence region projected onto the MTP

The propagation of a multidimensional confidence region requires a computational effort much larger than the onedimensional case. A large number of subdomains is created, typically in the order of thousands, and many of them reach the maximum number of splits. This has a significant effect on the reliability of the method, since the accuracy of the final map is in general compromised. When a subdomain reaches $\mathcal{N}_{\text {max }}$, if the nonlinearities are large or if a long integration is performed, the accuracy of the polynomial is totally lost. Even in the best case, in wich the post- $\mathcal{N}_{\max }$ integration is 
very short and therefore the polynomial maintains a certain accuracy, the dimension of the subdomains can be too large for a proper estimation of impacting surface. In general this means that the assumption that a single subdomain is either a full impactor or completely outside of the Earth may be too inaccurate for a proper IP estimation. The technique adopted to overcome this issue is now presented.

During the integration, the distance from the Earth is checked not only at the center but also at four points on the boundary of each subdomain. Instead of considering the four corners of the box, the evaluation points are taken as $(-1,0)$, $(1,0),(0,-1)$ and $(0,1)$. This choice is due to the fact that the four corner points are the most sensitive to the inaccuracy of the high-order polynomial coefficients. All the subdomains that have at least one point below the Earth surface are saved for further analysis. When the propagation of all boxes is over, the impacting boxes are retrieved and, if they belong to the group of subdomains that reached $\mathcal{N}_{\text {max }}$, they are reinitialized and repropagated from the last integration step at which the polynomial accuracy was met. This allows to obtain a further splitting only of the relevant boxes as depicted in Figure 14

Such repropagation is performed with a lower $\mathcal{N}_{\max }$, that has to be tuned in a compromise between accuracy and computational effort. In the multidimensional case, the boxes are prone to many splits and may reach $\mathcal{N}_{\text {max }}$ even in the second propagation, that means that they are split 32 times (considering $\mathcal{N}_{\text {max }}=16$ ) and still the polynomial accuracy is too low. Yet, their dimensions would be extremely small with respect to what is needed for an accurate IP computation and their propagation would require an extremely large computational effort. Instead, if the maximum number of splits is divided by two or four, the computational time reduces to values in the order of minutes, making the approach feasible.

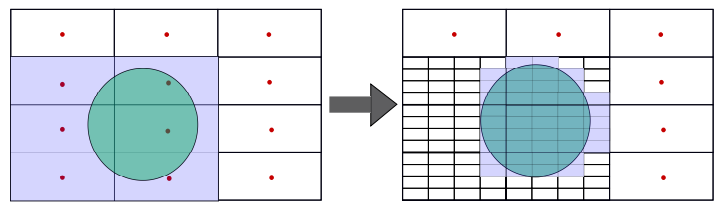

Fig. 14: Principle of the repropagation of the impacting boxes

The IP computation for the $2 \mathrm{D}$ tool is performed by assigning a two-dimensional Gaussian pdf to the initial domain and integrating it over the impacting domains. The standard expression of a 2D pdf:

$$
\operatorname{pdf}\left(x_{1}, x_{2}\right)=\frac{1}{2 \pi \sqrt{|\operatorname{det} \boldsymbol{\Sigma}|}} e^{-\frac{1}{2}(\mathbf{x}-\boldsymbol{\mu})^{T} \boldsymbol{\Sigma}^{-1}(\mathbf{x}-\boldsymbol{\mu})}
$$

is simplified thanks to the fact that the pdf is associated to the space of the eigenvectors of the initial domain and therefore the covariance matrix $\boldsymbol{\Sigma}$ is diagonal:

$$
\operatorname{pdf}_{\mathrm{i}}\left(\delta_{1_{i}}, \delta_{2_{i}}\right)=\frac{1}{2 \pi \sqrt{\sigma_{1}^{2} \sigma_{2}^{2}}} e^{-\frac{1}{2} \delta_{1_{i}}^{2} 3^{2}-\frac{1}{2} \delta_{2_{i}}^{2} 3^{2}}
$$

with the same nomenclature as in Equation 28 and where 3 at the exponent represents the sigma-interval of the analysis: $[-3 \sigma,+3 \sigma]$.
In a first approximation approach, all the impacting boxes resulting from the first ADS propagation are considered as full impactors. The 2D probability density is obtained by evaluating Equation 35 at the center, i.e. $\delta_{1_{i}}=\delta_{2_{i}}=0$ and their area is simply computed as:

$$
\mathcal{A}_{i}=3 \sigma_{1} 3 \sigma_{2} w_{1_{i}} w_{2_{i}}
$$

The impact probability is therefore computed by:

$$
I P=\sum_{i} \operatorname{pdf}_{i} \mathcal{A}_{i}
$$

This result may be strongly affected by the fact that the impacting subdomains have reached $\mathcal{N}_{\max }$ and therefore neither they can be reliably assumed as full impactors nor they can be used for a computation of the exact intersection. The impactors repropagation, presented in the previous section, can provide the necessary refinement. In this case the impacting area is computed as:

$$
\mathcal{A}_{i}=\mathcal{G}_{i} 3 \sigma_{1} 3 \sigma_{2} w_{1_{i}} w_{2_{i}}
$$

where $\mathcal{G}_{i}$ is a coefficient that specifies the portion of the box that really collides with the Earth and is evaluated as:

$$
\mathcal{G}_{i}=\sum_{j} \frac{1}{2} w_{1_{j}} \frac{1}{2} w_{2_{j}}
$$

where $w_{1 j}$ and $w_{2_{j}}$ are the widths in the two dimensions of the $j$-th sub-subdomain generated by the repropagation of the i-th subdoman that had a collision with the Earth surface.

The results obtained with the approximated and refined methods are listed in Table 3 The repropagation of the impacting boxes, in this case with $\mathcal{N}_{\max }=4$, requires about 3 additional hours of computational time but the outcome in terms of IP accuracy is much more satisfactory if compared to the IP value provided by NEODyS.

A delicate aspect of this procedure is the choice of $\mathcal{N}_{\max }$ for the repropagation of the first impactors. In this case $\mathcal{N}_{\text {max }}=4$ was a very good choice but it may be too low. In fact, in cases where the real impact probability is very small or the dynamics is highly nonlinear the actual impactors may represent a very tiny region of the impacting subdomains of the first propagation and therefore a higher value for $\mathcal{N}_{\text {max }}$ may be required, still considering that as this value grows, the computational effort may significantly rise.

\begin{tabular}{cccc}
\hline & comp. time $[\mathrm{h}]$ & 2D IP & IP $_{\text {NEODyS }}$ \\
\hline not refined & 8.48 & $5.8983 \cdot 10^{-4}$ & $4.10 \cdot 10^{-5}$ \\
refined & 11.51 & $4.3003 \cdot 10^{-5}$ & $4.10 \cdot 10^{-5}$ \\
\hline
\end{tabular}

Tab. 3: 2016 LP10 impact probability computation

\section{CONCLUSION}

This paper presented a differential algebraic approach to the Line of Variations technique to compute the impact probability of NEOs. The presented technique makes use of an automatic domain splitting algorithm to provide an accurate polynomial map from the initial to the final state during the 
numerical propagation. When the impactors are identified, the exact intersection between the LOV and the Earth surface can be computed, provided that the polynomial map is accurate enough such as in a direct encounter case. In the case of a resonant return, the nonlinearities of the dynamics enlarge the uncertainty and the final polynomial map is inaccurate. To correct this issue, the inaccurate subdomains of interest are repropagated and finally the impact probability is computed.

The key role of the eigenvalues ratio is highlighted to determine if the pure one-dimensional technique is reliable or more dimensions shall be included in the analysis. While the generation and propagation of a $2 \mathrm{D}$ plane of variations is straightforward within DA, the identification of the impactors and the computation of the IP presents some complexity. The technique developed so far is computationally expensive, yet it offers the possibility to estimate the impact probability for every possible configuration of the initial confidence region.

The method presented in this paper does not overcome the standard LOV technique in reliability or accuracy but it paves the way to significant developments. The application of DA to this type of uncertainty propagation has been proved to be valuable. The optimization of the propagator and the development of a fully automated algorithm would provide a reliable alternative to the standard technique used today.

\section{Bibliography}

[1] M. Berz, Advances in imaging and electron physics, 1999, Academic Press

[2] S. R. Chesley, P. W. Chodas, Asteroid close approaches: analysis and potential impact detection, Asteroids III, 55 (2002), University of Arizona Press

[3] P. Di Lizia, R. Armellin, M. Lavagna Application of high order expansions of two-point boundary value problems to astrodynamics. Celestial Mechanics and Dynamical Astronomy, 2008, 102.4: 355-375.

[4] A. Milani, The asteroid identification problem: I. recovery of lost asteroids, Icarus, 137-2 (1999), 269-292, Elsevier

[5] A. Milani, G. B. Valsecchi, The Asteroid Identification Problem: II. Target Plane Confidence Boundaries, Icarus, 140-2 (1999), 408-423, Elsevier

[6] M. Rasotto, A. Morselli, A. Wittig, M. Massari, P. Di Lizia, R. Armellin, C. Valles, G. Ortega Differential algebra space toolbox for nonlinear uncertainty propagation in space dynamics, 2016

[7] J. F. Ritt, Differential equations from the algebraic standpoint, 14 (1932), American Mathematical Soc.

[8] M. Valli, R. Armellin, P. Di Lizia, MR. Lavagna Nonlinear mapping of uncertainties in celestial mechanics, Journal of Guidance, Control, and Dynamic, 36-1 (2012), 48-63, American Institute of Aeronautics and Astronautics
[9] A. Wittig, P. Di Lizia, R. Armellin, K. Makino, F. Bernelli-Zazzera, M. Berz Propagation of large uncertainty sets in orbital dynamics by automatic domain splitting, Celestial Mechanics and Dynamical Astronomy, 122-3 (2015), 239-261, Springer

[10] D. K. Yeomans, P. W. Chodas, Predicting close approaches of asteroids and comets to Earth, Hazards due to Comets and Asteroids (1994), 241-258, University of Arizona Press 